\title{
Transient and recurrence processes in open system
}

Vladimirsky Eduard Iosifovich*, Ismailov Bahram Israfil

Department of Instrumentation Engineering, Azerbaijan State Oil and Industrial University, Baku, Azerbaijan

\section{ARTICLE IN F O}

\section{Article history:}

Received 18 May 2017

Received in revised form

11 August 2017

Accepted 20 August 2017

Keywords:

Poincare recurrence

Generalized memory

Tsallis entropy

Fractional hyperchaotic systems

\section{Introduction}

In most cases of new effects discovered in recent years include the research processes in nonlinear dissipative systems and environments. Problems outlined in the book will be considered in the context of modern scientific field - physics of open systems (Marwan et al., 2007; Ibedou and Miyata, 2008; Butkovskiy et al., 2013; Grigorenko and Grigorenko, 2003).

Noteworthy new features of so-called reversible mappings that allow for new look into the physical processes in the chaotic and stochastic systems.

It is known that the dynamic system with complex trajectories character can be described in terms of the geometry of limit sets in the phase space, as well as the evolution of the phase trajectories in time. Feature of the temporal dynamics of reversible is the so-called Poincare return, meaning that any trajectory, starting from a point $x_{0}$ of the phase space, eventually an infinite number of times will pass arbitrarily close to the initial conditions.

Depending of the system operation mode return will either (in the motion is stable periodic) or quasiperiodic (driving in $n$-dimension of the torus), or be a random sequence of time $\tau_{k}=t_{k+1}-t_{k}$ when $t_{k}$ corresponds to time path to enter the $\varepsilon$ neighborhood of $x_{0}$. So, for the first time of chaotic attractor's limited return: $\tau_{k}=t_{k+1}-t_{k}<z$ for all $k=1,2, \ldots$, which is a consequence of the presence of

\footnotetext{
* Corresponding Author.

Email Address: Eduard.Vladimirsky@hotmail.com (V. E. Iosifovich)

https://doi.org/10.21833/ijaas.2017.010.015

2313-626X/C) 2017 The Authors. Published by IASE.

This is an open access article under the CC BY-NC-ND license

(http://creativecommons.org/licenses/by-nc-nd/4.0/)
}

\begin{abstract}
The results of studies of transient and recurrent processes in open systems are shown. Questions related to the features of fractional systems dynamics of the studied processes, we propose a generalized Poincare repetition of Poincare. Developed a mathematical model of the transition
\end{abstract}

C 2017 The Authors. Published by IASE. This is an open access article under the CC BY-NC-ND license (http://creativecommons.org/licenses/by-nc-nd/4.0/).

a minimal set in the system. Movement of the attraction satisfied specified properties, Poincare called Poisson stable.

It continues to be the actual problem analyses and synthesis of a large number of interacting heterogeneous information flow in complex structures. In the process of evolution, in an open system, increase information flows and objects leads to a complication of information components that causes a buildup of chaotic processes, which translate the system into a state of dynamic chaos.

Wherein the system produces a new random information, the rate of this process is the higher the greater the degree of randomness. In this important to keep track of Poincare return time as the main indicators and characteristics, show the dynamics of the system in time repeatability. Great interests are the processes of mixing multidimensional heterogeneous system. In this context, attention is drawn to the processes of mixing multidimensional heterogeneous systems. By mixing multidimensional systems may be coherent structures that require analyses and evolution on this structure.

The resonance caused works by Lekien et al. (2007), Shadden (2011), and Kusch and Ottino (1992) devoted to the study of coherent Lagrange structures, which are ranges of fields of finite-time Lyapunov exponent (FTLE). These ranges can be considered as a finite-time mixed formation. Concept of this work is applicable to flows with random timedependent and in particular, flows which are defined in a finite-time interval. This problem is further updated in the analyses of nonlinear mixed physical systems, in which examples of Lagrangian coherent structures are stable and unstable manifolds of fixed points and periodic orbits. Along with the mixing process occurs problem of mixed transport flow. To their arises paradigm consideration of analysis and 
synthesis of structure as the "mixing-transportcontrol" of nonlinear physical processes.

It should be noted that the asymptotic distribution of Poincare recurrences is exponential for a wide class of mixing system, even if they uniformly hyperbolic.

Some preliminary investigations show that at least for the skew and for the mixing return times spectra also hold for the successive Poincare recurrences. Currently, there is intensification of research into the processes and phenomena, characterized by nonlocality, nonmarcov, hereditarily, fractality, nonhamiltonian. It is also paid great attention to studying the degree of non-locality and the power of long-term memory. Are mathematical methods of one of the modern areas of theoretical physics- fractional dynamics. This is especially true when it comes to fractal structure systems. It is important to note that the description of the properties of systems with fractal structure cannot be used Euclidean representation geometry. There is a need to analyze these processes in terms of fractional geometry (Nigmatullin, 1992).

Systems with fractal feature characterized by such effect as memory, complex spatial processes of mixing and self-organization. Thus formed a new scientific field-physic of open systems, in which the combined areas such as synergy, dissipative structures, deterministic chaos, fractional dynamics in the various branches of science. Methods of integral-differential fractional and fractional calculus, a history spanning more than three hundred years, back to the research of prominent mathematicians, such as Riesz (1949) and Brox (2017).

New opportunities in mathematics and theoretical physics in the open when the order of $\alpha$ differential operator $D_{x}^{\alpha}$ becomes arbitrary parameter. Here fractional derivative index allows to consider features of open systems. The book uses fundamental research of Chirikov (1979), Zaslavsky (2002), Tarasov (2010), and others.

In spite of the anomalous chapter, the consideration is in terms of Poincare return time. Important practical problem when working with nonlinear systems is their discrete in the time mapping, which allows to conclude that the nature of the continuous flow.

In addition, the book discusses issues of transient in multidimensional chaotic systems of fractional order and offered nontraditional chaotic and stochastic filters, the base of which is integrative component of the average Poincare return time.

Above actualizes the problem of the research of transients in multidimensional chaotic and stochastic systems of fractional-order. It should be noted there is a reassessment role of chaos in the process of evolution of nonlinear multidimensional systems. Noted that the chaos is necessary for the system output to one of the possible attractors; chaos is at the heart of combining mechanisms simpler structures in complex, and finally can act as a system of behavior change regimes.
It is important to note the features of a collective of multidimensional processes and phenomena in the fractional chaotic systems, in the context of the observed transients.

A big role in the description of the behavior of open systems plays a synergetic view of its evolution as a whole that is in terms of attractors, transition states, stability, bifurcations, of dynamical chaos and other. A basic element of his research is to follow the phase portrait and its changes when you change the synergetic model parameters.

From the position of mathematics synergetic aspect of the process of evolution is a change in the topological structure of the phase space of an open system.

Tracking change this structure, as the transition process requires the formation of a generalized criterion that recognizes entering the system in one or another state.

It is known that transient is a process whose parameters vary over time. Bat it should be noted that transition may occur both in domestic and external perturbation. The above describes the structure of the research multidimensional chaotic, stochastic and kinetic fractional-order systems (Fig. 1). Implementation of such a frame work should be based on some of the main provisions.

It should be noted that deviation of the state of a synergetic system from the position of thermodynamics equilibrium is accompanied by development of chaotic, stochastic transients, destroying properties of ergodicity, additiveness and local equilibrium; entropy reduction; fractional structure and search for a new stability state. Important it is regard is the use of Tsallis entropy.

Moreover the implementation of the tasks on the stability of multidimensional chaotic systems of fractional order can manifest hidden oscillation, that are not established after the transition process from the neighborhoods of the stationary states. Here a simple simulation can lead to erroneous results.

Therefore, numerous results dealing with mechanisms of the generation of attractors, their localization in the phase space, and the evolution of their characteristics where obtained with the use of computer modeling well- known examples of the existence of hidden attractor in multidimensional models of automated control systems are given by counterexamples to the Vaidyanathan and Volos (2017) conjecture, where the unique stable-in-small equilibrium co-exists with an orbital stable cycle. Thus, the control problem is the development a mathematical model studies of transient and recurrent processes in the multi-dimensional chaotic systems of fractional order.

Significant role information the trends play an entropy oscillation that arising near the steady state systems (Liu and Teel, 2014).

Treads can be mathematically represented the inequalities or sign extremum. In addition to this group of laws include equilibrium conditions, stability criteria, the criteria of evolution, the 
minimum entropy production theorem, the law of increasing entropy, and others (Liu and Teel, 2014).
With regard to the entropy, it can only be changed in irreversible processes. Reversibility is characteristic for conservative system.

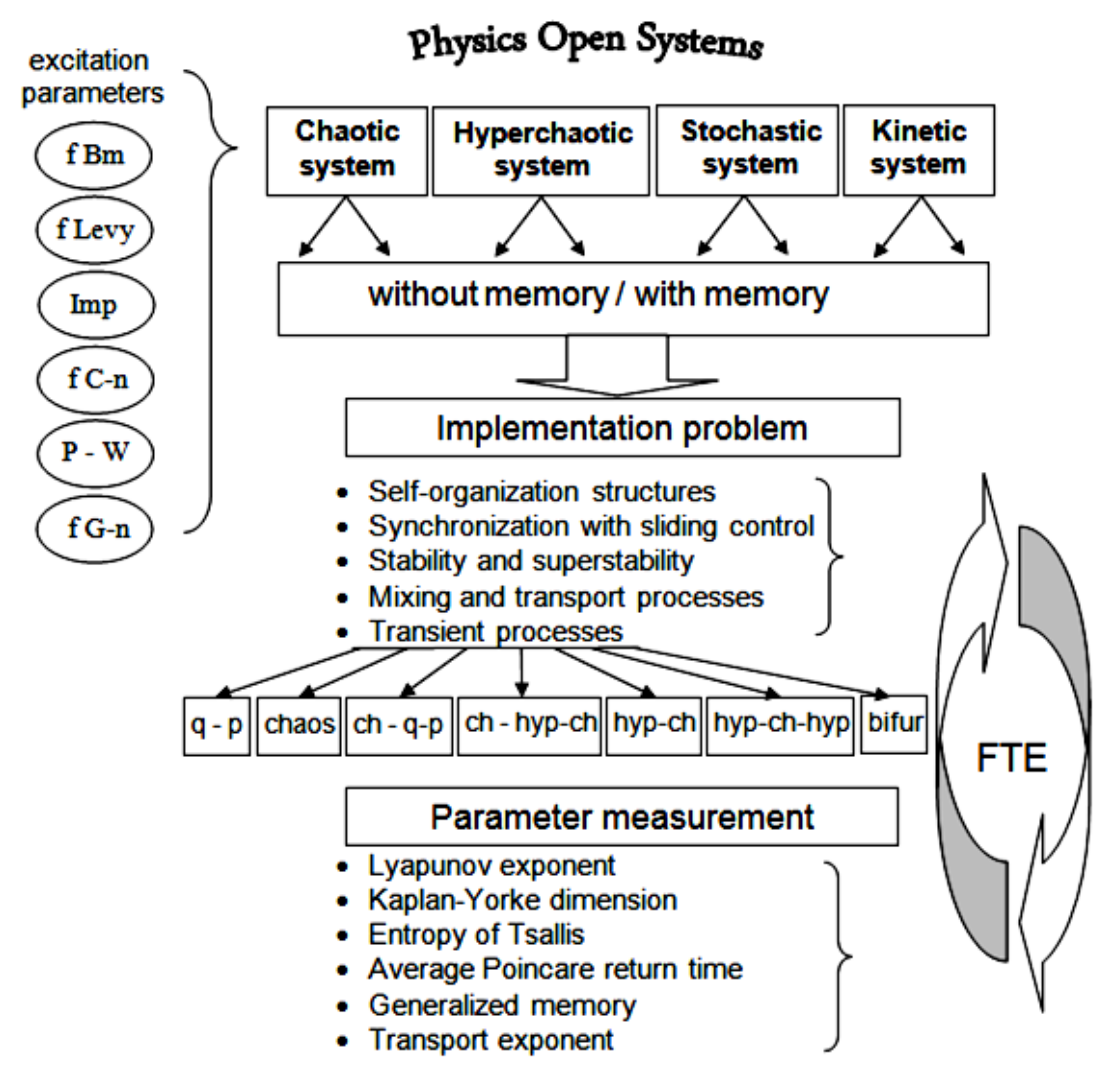

$\mathbf{f B m}$ - fractional Brownian motion, fLevy - fractional Levy motion, Imp - impulsive function, fCN - fractional Colored noise, P-W - piecewise function, fGn fractional Gaussian noise, $\mathbf{q}$ - p - quasi-periodic, ch - q-p - chaos - quasi-periodic, ch-stoch - chaos - stochastic, ch - hyp-ch - chaos - hyper-chaos, hyp - ch hyp - hyper-chaos - chaos - hyper-chaos, bifur - bifurcation. F T E - fractional time evolution

Fig. 1: The investigated problems of chaotic systems, the interference components affecting them, the development of processes, and the measured parameters

In such systems, entropy is always constant and self-organizing them is not possible. Philosophical concept of recover the whole of nature to the state it was the beginning of the process (Liu and Teel, 2014).

\section{Basic provisions}

\subsection{Recurrence Poincare}

In the 1880s Henri Poincare had obtained a number of important results, which formed the basis of the modern theory of dynamical systems.

In particular, he noted the complexity of the behavior of the system in the vicinity of the so-called homoclinic trajectory (trajectory tends to a fixed point or a periodic trajectory with both $\rightarrow \infty$, and when $t \rightarrow-\infty$ ) (Nigmatullin, 1992), it was published in 1890, as the "recurrence theorem" (Marwan et al., 2007).

This theorem is the basis of the modern of measure preserving transformations, known as the ergodic theory.

Let $X$ open area in $n$-dimensional space with a homeomorphism $T$ for $x$ yourself, keep the volume. With repeated MSE $T$ of any point $x$ generates a sequence, $T x, T^{2} x, \ldots, T_{(x)}^{i}, \ldots$, called positive semi orbit $x$. When $x \in G$ for any infinity set of positive values ..., we speak of a recurring point $x$ of an open set $G \subset X$.

On a content level Poincare theorem states for any open set $G \subset X$ points, returning relatively, are all points $G$, except for some set of the first category measure zero. Formally takes place

Theorem 1: Let $T$ - be a measure-preserving transformation of a probability space $(X, \mu)$ and let $A \subset X$. be a measurable set (Marwan et al., 2007). Then for any natural number $N \subset N$

$A\left(\left\{x \in A:\left\{T^{n}(x)\right\}_{n \geq N} \subset(X \backslash A)\right\}\right)=0$

where $T$ - recurrence time; $X$ - arbitrary measurable set; $\mu(\cdot)$ - probability measure; $x$ - parameter of normalized number; $N$ - set of natural numbers. Short proof of this theorem in (Marwan et al., 2007).

\subsection{Topological order space}

Definition 1: The number is called as a metric order of a compact $A$

$k=\lim \left(-\ln N_{A}(\varepsilon) / \ln \varepsilon\right)$

where $\varepsilon$ - the sphere of radius $\varepsilon ; N(\varepsilon)$ - number of spheres in a final sub covering of a set. 
The lower bound of metric orders for all metrics of a compact $A$ (called by metric dimension) is equal his Lebesgue to dimension.

However it appeared that the metric order entered in Liu and Teel (2014) coincides with the lower side the fractal dimension of HausdorffBezikovich defined in the terms "box-counting". Takes place:

Theorem 2: For any compact metric space $X$ (Ibedou and Miyata, 2008).

$\operatorname{dim} X=\inf \left\{\frac{\lim }{\varepsilon \rightarrow 0} \frac{\log N_{\varepsilon, d}(X)}{-\log \varepsilon}:\right.$ d is a ametric on $\left.X\right\}$

where

$N_{s, d}(X)=$

$\min \{|U|: U$ is a finiteopen covering $X$ with mesh $\leq \varepsilon\}$.

From here $\left(X, d_{f}\right)$,- compact fractal metric space with dimension $d_{f}$.

Here it is important to note that at the description of properties of systems with fractional structure it is impossible to use representation of Euclidean geometry. There is a need of the analysis of these processes for terms of geometry of fractional dimension.

Remark: Butkovskiy et al. (2013) presented results of communication of a fractional integrodifferentiation (in Riemann-Liouville or Gryunvalda-Letnikov's terms) with Koch's curves.

It is noted that biunique communication between fractals and fractional operators does not exist: fractals can be generated and described without use of fractional operations, and defined the fractional operator not necessarily generates defined (unambiguously with it connected) fractal process or fractal variety.

However use of fractional operations allows generating other fractal process (variety) which fractal dimension is connected with an indicator of a fractional integrodifferentiation a linear ratio on the basis of the set fractal process (variety). Butkovskiy et al. (2013) fractional integrals of Riemann Liouville are understood as integrals on space of fractional dimension.

Thus the indicator of integration is connected with dimension of space an unambiguous ratio.

In this regard consideration of dimension of chaotic systems of a fractional order causes interest. So, Grigorenko and Grigorenko (2003) was noted that dimension of such systems can be defined by the sum of fractional exponents $\Sigma$, and $\Sigma<3$ is the most effective.

Let the chaotic fractional system of Lorentz take place (Vladimirsky and Ismailov, 2016):

$\frac{d^{\alpha}}{d t^{\alpha}} x=\sigma(y-x) \frac{d^{\beta}}{d t^{\beta}} y=\rho x-y-x z^{\prime} \frac{d^{\gamma}}{d t^{\gamma}} z=x y-b z$ here

$\sigma=10, \rho=28, b=8 / 3 ; 0<\alpha, \beta, \gamma \leq 1, r \geq 1$.

Then fractional dimension of system of the equations will have an appearance (Grigorenko and Grigorenko, 2003):

$\alpha+\beta+\gamma=\Sigma$.

So, for example, for Lorentz's system with fractional exponents $\alpha=\beta=\gamma=0.99$, effective dimension $\Sigma=2.97$.

This, in the context of fractional dynamics let $\tilde{X}$ any set of nonlinear physical systems, $A^{\alpha}$ - a subset of a set $\tilde{X}$ of systems of a fractional order with memory $A^{\alpha} \subset \tilde{X}$. Then a triad $\left(\tilde{X}, A^{\alpha}, \Sigma\right)$ - compact fractional metric space with dimension $\Sigma$.

Let's designate $W \in\left(X, d_{f}\right)$ on the basis and Remarks $\left(\tilde{X}, A^{\alpha}, \Sigma\right) \subset W$ (Nigmatullin, 1992).

Let's consider transformation $W$ at an angle of communications of average time of return of Poincare $\langle\tau\rangle$ with $d_{f}$ and "residual" memory $(t)$. Here:

$g:\langle\tau\rangle \Rightarrow d_{f} l: d_{f} \Rightarrow(t) \chi:\langle\tau\rangle \Rightarrow(g, l)$

From here $U \in(X,\langle\tau\rangle)$ - the generalized compact metric space of Poincare with dimension $\langle\tau\rangle$.

\subsection{Generalized memory}

As said, the analysis and synthesis of multidimensional chaotic system of fractional-order there was a problem with memory estimation.

Axiomatic: Let the trajectory of the generalized memory system is of the form (Liu and Teel, 2014; Vladimirsky and Ismailov, 2015; Vladimirsky and Ismailov, 2016):

$Q_{G M}=Q_{\geq 0} \cup Q_{\leq 0}$

Definition 2: $Q_{\geq 0} \subset Q_{G M}$ - it called semi-trajectory to the trajectory $Q_{G M}$, if each $t>0$ it the inclusion

$Q_{\geq 0} \subset O_{\varepsilon}^{m e m} \cup_{j=1}^{j-1}\left(\left[t_{j}, t_{j+1}\right], j\right)$.

Here $Q_{\geq 0}\left[t_{j}, t_{j+1}\right]$ - segment of the semi-trajectory memory of responsible values $t \subset\left[t_{j}, t_{j+1}\right]$ and $O_{\varepsilon}^{\text {mem }}$ $\varepsilon$ - neighborhood of the corresponding set.

Definition 3: $Q_{\leq 0} \subset Q_{G M}$ It called semi-trajectory to the trajectory $Q_{G M}$, if each $t<0$ it the inclusion $Q_{\leq 0} \subset O_{\varepsilon}^{l m} \cup_{j=1}^{j-1}\left(\left[s_{k}, s_{k-1}\right],-k+1\right)$.

Here $Q_{\leq 0}\left[s_{k}, s_{k-1}\right]$ - segment of the semi-trajectory "loss memory" of responsible values $\in\left[s_{k}, s_{k-1}\right]$ and $O_{\varepsilon}^{l m}-\varepsilon$ - neighborhood of the corresponding set.

Definition 4: Semi-trajectory $Q_{\geq 0} \subset Q$ recurrent if for every $t>0$ inclusion 
$Q_{\geq 0} \subset O_{\varepsilon} \cup_{j=1}^{j-1}\left(\left[t_{j}, t_{j+1}\right], j\right)$.

Here $Q_{\geq 0} \subset\left[t_{j}, t_{j+1}\right]$ - segment of the semi-trajectory recurrent of responsible values $t \subset\left[t_{j}, t_{j+1}\right]$.

Definition 5: Semi-trajectory $Q_{\leq 0} \subset Q$ not recurrent if for every $t<0$ it the inclusion

$Q_{\leq 0} \subset O_{\varepsilon} \cup_{k=1}^{k}\left(\left[s_{k}, s_{k-1}\right], k+1\right)$.

Remark: It is known the average return time of Poincare is determined by the fractal dimensions the trajectories of generalized memory, GM. Hence the memory loss will be determined by the difference between global and local fractal dimensions, which means that the recurrence and no-recurrence semitrajectories respectively.

\subsection{Formation of loss memory}

It is a known that during the Poincare recurrence characterizes as a "residual", and the real memory of the fractional-order system. Hence the equivalence between the spectrums of the Poincare returns time and distribution of generalized memory.

Los memory is determined by the difference between the global and the local fractal dimensions, which means, respectively, reversible and irreversible processes. Loss of information numerically defines the entropy.

\subsection{The spectrum of the dimensions of the Poincare returns times}

From the perspective of mathematics synergetic aspect of the process of evolution is a change of a topological structure of the phase space of an open system.

Tracking change this structure as transient process requires the formation of a generalized criterion for recognizing the system in a certain state of chaos-quasi-periodic-hyper chaos and so on.

In the work, as a generalized criterion proposed spectrum of dimensions Poincare return time, describing how geometric, information, and dynamics characteristics of the transient process. That is generalized criterion is formed on the basis of synergetic principles. Spectrum of dimensions for the Poincare return time is a functional dependence $\widetilde{\alpha_{q}}$ dimension type characteristics from the scale $q$ expressed in terms of the Hausdorf dimension $\operatorname{dim}_{H}$ (Eq. 1) (Afrajmovich et al., 2011):

$\widetilde{\alpha_{q}}=\operatorname{dim}_{H}(x)(1-q / h)$,

where $q$ - scale, $x$ - invariant set, $h$ - topological entropy.

Weng et al. (2015) Showed that the transport exponent $\mu$ defined by the relationship $\lim h(\mu)=$ $h\left(d_{f}\right)$ and $\lim h(\mu)=h\left(d_{f}+1\right)$ respectively. Noted that $\mu^{\text {opt }}$ defined as (Eq. 2) (Weng et al., 2015):

$\mu^{o p t} \approx d_{f}+2-\frac{\log (2)}{2\left(\log (2)+3+d_{f}\right)}$ where $d_{f}$ - fractal dimension. However, in practical, is used (Eq. 3)

$\mu^{o p t} \approx d_{f}+2$

In addition, knowing that $h \leq \sum_{\lambda_{i>0}} \lambda_{i}$, where $\lambda_{i^{-}}$ Lyapunov exponents, spectrum dimensions of the Poincare return time will look (Eq. 4):

$\widetilde{\alpha_{\tilde{q}}}=d_{f}(X)\left\{(1-q / h)(\mu-2)\left(Q_{\geq 0}\right)(h(\lambda))\right\}$

Thus, there is functional dependence of the memory of the transport exponent mixing heterogeneous chaotic maps, as well as the Lyapunov exponent.

\subsection{Tsallis entropy}

The interaction of complex heterogeneous chaotic fractional systems caused a revision of traditional Boltzmann entropy estimation.

Attempt of this review contained in thermodynamics Tsallis, which leads to a statistical physics not Boltzmann type: individual particles with Boltzmann statistics + strong interactions $\Rightarrow$ new degrees of freedom with no Boltzmann statistics + lack of interaction. Tsallis the entropy has the form (Eq. 5) (Tsallis and Tirnakli, 2010):

$S_{\tilde{q}}=-\sum_{i}\left(P_{i}^{\tilde{q}} \ln _{g}\left(P_{i}\right)\right)=\left(1-\sum_{i} P_{i}^{\tilde{q}}\right) /(\tilde{q}-1)$

where $\tilde{q}$ - measure is not extensiveness of the system and can take an values: $-\infty:+\infty, i$ - systems status number, $P_{i}$ - the probability of finding the system in state $i, \Sigma_{i}$ - summing over all states.

Then the entropy oscillations in the segment $Q_{\leq 0}$ of memory loss will be determined by the entropy Tsallis $S_{\tilde{q}}$.

\section{A mathematical model of transition processes}

In this section seems paradigm transience/recurrence/stability for multidimensional chaotic and stochastic systems of fractional order. Here is an important problem is on formation of the axioms that reflect this paradigm. A quick lock at the various systems in the context of this paradigm. Basic principles and definitions:

\subsection{Generalized transition state theory (TST)}

Definition 6: Any form of TST, such as micro canonical variation TST, canonical variation TST, and improved canonical variation TST, in which the transition state is not necessarily located at the saddle point, is referred to as generalized transition. Formally have the following:

Definition 7: We say that $X$ is recurrent if $\lim _{\inf _{t \rightarrow \infty}}\left\|X_{t}\right\|=0$ a.s. We say that $X$ is transient if $\lim \inf _{t \rightarrow \infty}=0$ a.s. 


\subsection{The transient signal model}

Definition 8: A transient signal $x_{d}[n]$ is formed as linear combination of a finite number $d$ of real decaying exponentials with the structure (Eq. 6) (Lahlou and Oppenheim, 2014):

$x_{d}[n]=\sum_{k=1}^{d} \lambda_{k}\left(\rho_{k}\right)^{n}, \rho_{k} \neq 0,-1<\rho_{1}<\cdots<\rho_{d}<1$

for all $n \geq 0$.

Here $\alpha=\left\{\alpha_{k}\right\}_{k=1}^{d}$ - amplitude coefficient, $x_{d}=\left\{x_{d}[n]\right\}_{n=0}^{N-1}$ - parameter sequence,

$\rho=\left\{\rho_{k}\right\}_{k=1}^{d}$-attenuation signal.

\subsection{The discrete transient transforms}

Definition 9: The Discrete Transient Transform (DTT) $X[k]$ and inverse DTT (IDDT) of a general length $N$ sequence $x[n]$ are defined as (Eqs. 7 and 8) (Lahlou and Oppenheim, 2014):

$X[k]=\sum_{n=0}^{N-1} x[n] \psi_{k}[n], \quad 1 \leq k \leq N$

$x[n]=\sum_{k=1}^{N} X[k] \varphi_{k}[n], \quad 0 \leq n \leq N-1$

where $\varphi$ is a real exponential basis and $\psi$ is the corresponding dual basis. Recurrence and Transience in Markov chain (Norris, 1998).

Definition 10: Let $\left(X_{n}\right)_{n \geq 0}$ be a Markov chain with transition matrix:

We say that a state $i$ is recurrent if

$P_{i}\left(X_{n}=i\right.$ for infinitely many $\left.n\right)=1$

We say that $i$ is transient if

$P_{i}\left(X_{n}=i\right.$ for infinitely many $\left.m\right)=0$

Thus a recurrent state is one to which you keep coming back and a transient state is one which you eventually leave for ever. Norris (1998) showed the study of these states. For stochastic systems have the following

Definition 11: Brownian motion $\{B(t): t \geq 0\}$ is (Teo, 2012):

i. transient if $\lim _{t \rightarrow \infty}|B(t)|=\infty$ a.s.

ii. point recurrent if a.s. for every $x \in R^{d}$, there is an increasing sequence such that $B\left(t_{n}\right)=x$ for all $n \in$ $N$.

iii. neighborhood recurrent if a.s. for every $x \in$ $R^{d}$, and $\varepsilon>0$, there exists an increasing sequence $t_{n}$ such that $B\left(t_{n}\right) \in B_{\varepsilon}(x)$ for all $n \in N$.

Thus Altmann and Tél (2008) obtained a description on the Poincare recurrences of chaotic systems in terms of the ergodic theory of transient chaos.

Hybrid fractional noise: The sawtooth wave, called the "castle rim function" by Trott is the periodic function (Eq. 9):
$S(x)=\operatorname{Arac}\left(\frac{x}{T}+\varphi\right)$

where $\operatorname{frac}(x)$ is the fractional part $\operatorname{frac}(x) \equiv x-$ $[x], A$ is the amplitude, $T$ is period of the wave, and $\varphi$ is its phase. Here

$\operatorname{frac}(x) \equiv\left\{\begin{array}{l}x-[x] x \geq 0 \text { celling function } \\ x-[x] x<0 \text { celling function }\end{array}\right.$

Fractional order colored noise: The integer order time-correlated (colored) noise is given by the following relation (Eq. 10) (Norris, 1998):

$\mu_{k+1}=\omega_{k} \mu_{k}+\omega_{k}$

where $\mu_{k} \in R$ is a time-correlated noise, $f^{\prime} \in R$ is a parameter of this noise, and $\omega_{k} \in R$ is an uncorrelated noise, for example, white Gaussian noise. This can be rewritten in the difference from (Eq. 11) (Sierociuk and Ziubinski, 2014):

$\Delta_{\mu_{k+1}}^{1}=f \mu_{k}+\omega_{k}$

where $f=f^{\prime}-1$.

The relation can be generalized onto a fractional order time-correlated noise. The colored fractional order noise is given as follows (Eq. 12) (Sierociuk and Ziubinski, 2014):

$\Delta_{\mu_{k+1}}^{\alpha}=f \mu_{k}+\omega_{k}$

where in this, case $\mu_{k}$ is a fractional colored noise, $\alpha$ is an order of the noise and $\omega_{k}$ is an uncorrelated noise. This equation is a discrete equivalent of fractional differential equation witch describes dynamics of the correlated noise. The hybrid scheme of fractional noise given as follows (Eq. 13):

$N=S(x) \cup \Delta_{\mu_{k+1}}^{\alpha}$

Analysis of the above offers transient chaotic system of fractional-order and Poincare recurrence consider as the trajectories of generalized memory $Q$ is mapping to the spectrum of dimensions Poincare $\Psi$ (Eq. 14).

$\Psi^{\text {def }}=Q \Rightarrow \widetilde{\alpha_{q}}$.

Regarding stability it will be defined as local stability of Lyapunov semi-trajectories $Q_{\geq 0}$.

Further continue the recovery of the signal, followed by an analysis of his character that will be noted in section.

\subsection{Transient with recovery}

The implementation of the tasks on the stability of multidimensional chaotic systems of fractional order can manifest hidden oscillation, that are not established after the transition process from the neighborhoods of the stationary states. Here a simple simulation can lead to erroneous results. 
Therefore, numerous results dealing with mechanisms of the generation of attractors their localization in the phase space, and the evolution of their characteristics where obtained with the use of computer modeling well - known examples of the existence of hidden attractor in multidimensional models of automated control systems are given by counterexamples to the Vaidyanathan and Volos (2017) conjecture, where the unique stable-in-small equilibrium co-exists with an orbital stable cycle (Leonov and Kuznetsov, 2011).

Effectively verified conditions for the existence hidden orbital stable cycles in some class multidimensional systems were obtained in Leonov and Kuznetsov (2011).

In this regard Ismailov (2016) proposed structure of the "SSA-lifting scheme", produced a reconstruction signal.

\section{The example of the tasks of synchronization with the tracking control is the following}

Formulation of the tasks: Consider the following $\mathrm{n}$-dimensional-fractional-order chaotic system (Eq. 15) (Vladimirsky and Ismailov, 2015; Vladimirsky and Ismailov, 2016)

$D^{q} X=F\left(X, X_{0}, \theta\right)$

where $X=\left(x_{1}, x_{2}, \ldots, x_{n}\right)^{T} \in R^{n}$ denotes the $\mathrm{n}$ dimensional state vector of the original system; $X_{0^{-}}$ represents the system initial state, $q=$ $\left(q_{1}, q_{2}, \ldots, q_{n}\right)^{T} \in R^{n}$ is a set of fractional order of the original system, and $\theta=\left(\theta_{1}, \theta_{2}, \ldots, \theta_{D}\right)^{T} \in R^{D}$ is the value of original system parameters.

Let the fractional-order derivative of the function $f(t)$. In the Caputo sense is defined as Eq. 16 (Vladimirsky and Ismailov, 2015; Vladimirsky and Ismailov, 2016):

$D^{q} f(t)=J^{m-q} f^{(m)}(t)$.

Here, $q$ is the fractional order, $m$ is an integer that satisfies $m-1 \leq q<m, f^{(m)}(t)$ is the ordinary $m$ th derivative of, and $J^{\mu}$ is the Riemann -Liouville integral operator of order $\mu>0$, defined by Eq. 17 (Vladimirsky and Ismailov, 2015; Vladimirsky and Ismailov, 2016):

$J^{\mu} g(t)=\frac{1}{\Gamma(\mu)} \int_{0}^{t}(t-\tau)^{\mu-1} g(\tau) d \tau$,

where $\Gamma(\bullet)$ denotes the gamma function. A particularly important case in many engineering applications is $0<q<1$. In this situation, Eq. 16 together with Eq. 17 represented as Eq. 18

$D_{*}^{q} f(t)=\frac{1}{\Gamma(1-q)} \int_{0}^{t}(t-\tau)^{-q} f^{t}(\tau) d \tau$.

The operator $D_{*}^{q}$ is often called " $q$ th- order Caputo differential operator" and will be used throughout the paper.

Topological synchronization: Unlike traditional methods of synchronization in Vladimirsky and Ismailov (2016) and Afrajmovich et al. (2011) proposed the concept of topological synchronization of coupled chaotic systems.

Definition 12: Two systems are topologically synchronized, if Poincare return times behave a similar way.

Thus match the dimensions of these two systems- a necessary condition for the topological synchronization: well indicates the "average similarity". It is known that the synchronization feature is the preservation of a certain frequency: in this case, is the relationship between the Poincare return times. Invariance of these ratios, are the timing mode.

\subsection{Synchronization between the two fractional hyperchaotic systems}

In the general case, synchronization of chaotic fractional-order systems, united has the form Eq. 19 (Vladimirsky and Ismailov, 2015; Vladimirsky and Ismailov, 2016):

$\frac{d^{\alpha} X}{d t^{\alpha}}=f(x), \frac{d^{\alpha} Y}{d t^{\alpha}}=\hat{g}(Y)+U(t)$

where $\alpha$ - order of derivative, $\alpha \in(0,1]$, master's $X \in$ $R^{n}$ and slave $Y \in R^{n}$ systems; $f: R^{n} \rightarrow R^{n}$ and $\hat{g}: R^{n} \rightarrow R^{n}$ - vector fields master and slave systems. In the general case condition synchronization systems defined as: $U(t)=\left(u_{1}, \ldots, u_{n}\right)^{T}$, i.e. $\lim _{t \rightarrow \infty}\|X-Y\|$, where $\|\cdot\|$ - Euclidean norm.

\subsection{Topological control}

Consider the following general structure of the fractional-order nonlinear system under control (Eq. 20) (Vladimirsky and Ismailov, 2015; Vladimirsky and Ismailov, 2016):

$$
{ }_{0} D_{t}^{q} x(t)=f(x(t)+B u(t))
$$

where $\quad u(t)=\left[u_{1}(t) u_{2}(t) \ldots u_{m}(t)\right]^{T} \quad$ is $\quad m-$ dimensional input vector that will be used and following control structure will be considered for state feedback (Eq. 21):

$u(t)=u_{e q}(t)+u_{s w}(t)$

where $u_{e q}(t)$ is equivalent control and $u_{s w}(t)$ is the switching control of the system.

With regard to the task the topological control will be submitting a number of definitions.

Definition 13: The system topologically controllable if and only if coincides with $\hat{x}$ on the basis of the criterion metrics "proximity" Hausdorf.

Theorem 3: Let $E$ and $F$ is compact subset $R^{n}, \varepsilon>0$. Hausdorf distance $H(E, F)$ satisfies the relation. $H(E, F) \leq \hat{\varepsilon} \Leftrightarrow E \subset F+\hat{\varepsilon}$ and $F \subset E+\hat{\varepsilon}$,

where $\hat{\varepsilon}>0$ the allowable threshold. 
Synchronization, control and stability of fractional order and the context of the generalized memory: Common challenges in the implementation of topological synchronization and topological control are a base - definition, theorem and intelligent iterative algorithm.

Fractional-order iterative learning control scheme (Fig. 2) is given as (Vladimirsky and Ismailov, 2015; Vladimirsky and Ismailov, 2016):

$U_{k+p}^{(\alpha)}(t)=F\left(U_{k}(t), e_{k}(t)\right)$,

where $e_{k}(t)=Y_{d}(t)-Y_{k}(t)$.

Definition 14: Two systems topologically controllable if and only if they are synchronized topologically.

Remark: If you synchronize at the same time regularity at the exit system there is a situation called passive control.

Otherwise the usual iterative procedure for the organization of regular structure on the system output.



Fig. 2: The basic scheme of the algorithm used to make iterative training of the control system with $Y_{d}(t)$ being the trajectory, $U_{k}(t)$ and $Y_{k}(t)$ the input and output signals

\subsection{Poincare recurrence diagram}

Mapping the system on the two-dimensional square matrices $[N, N]$ and of formula:

$R_{i, j}^{m, \varepsilon} i=\theta\left(\varepsilon_{i}-\left\|x_{i}-x_{j}\right\|\right), i, j=1, \ldots, N, i \neq j, x \in U^{\prime}$

where $N$ - number of considered (examined) condition $x_{i}, \varepsilon$ - size of a neighborhood of a point $x$ at the moment $i ;\|\cdot\|$ - norm; $\theta(\cdot)$ - function of Heaviside.

\subsection{Topological stability of hyperchaotic-order systems}

Determine the stability of the zero solution on the system

$\frac{d x}{d t}={ }_{q} \widehat{\Omega}, \quad{ }_{q} \widehat{\Omega}=\left\{\omega_{n}\right\}_{n=0}^{N},{ }_{q} \widehat{\Omega} \in{ }_{q} \widehat{\psi}$.
Proposition: Let $G M \in U$ be structure of generalized memory. If there exist a differentiable observed $V: R^{n} \rightarrow R^{+}$such that the two following hold:

(i)- if trajectory will pass thought the point 0 , i.e. $\dot{V}(x) \leq 0$, the system is stable with $d_{f}(\langle\tau\rangle)$ and matches $G M$;

(ii)- if trajectory will pass below the point 0 , the system is asymptotically stable with $d_{f}(\langle\tau\rangle)$.

Let the two hyperchaotic fractional-order systems:

- Fractional-order Rabinovich-Fabrikant system following (Vladimirsky and Ismailov, 2016):

$$
\left\{\begin{array}{c}
\dot{x_{1}}=x_{2}\left(x_{3}-1+x_{1}^{2}\right)+\gamma x_{1} \\
\dot{x_{2}}=x_{1}\left(3 x_{3}+1-x_{1}^{2}\right)+\gamma x_{2}, \\
\dot{x_{3}}=-2 x_{3}\left(x_{1} x_{2}+\alpha\right) \\
\dot{x_{4}}=-3 x_{3}\left(x_{2} x_{4}+\delta\right)+x_{4}^{2}
\end{array}\right.
$$

where $=0.14, \gamma=1.1,-0.01 \leq \delta \leq 7650$.

- The fractional-order Chen system as follows (Vladimirsky and Ismailov, 2016):

$$
\begin{aligned}
& \frac{d^{\alpha} x}{d t^{\alpha}}=a(y-x)+w+N \\
& \frac{d^{\alpha} y}{d t^{\alpha}}=b x-x z+c y, \\
& \frac{d^{\alpha} x}{d t^{\alpha}}=x y-d z, \\
& \frac{d^{\alpha} w}{d t^{\alpha}}=y z+r w,
\end{aligned}
$$

where $a=35, b=7, c=12, d=3, r=0.5$ and $\alpha=$ $0.9, N$ - hybrid scheme of fractional noise.

Iterative learning algorithm for topological synchronization on schematic "master-slave" with white tracking control for fractional-order hyperchaotic systems shows in Fig. 3 (Vladimirsky and Ismailov, 2016; Afrajmovich et al., 2011).

Further, using the signal reconstruction algorithm of the scheme "SSA-lifting scheme", we get a complete picture of the process of transition hyperchaos-chaos-chaos (Fig. 3).

Thus, there is a transient "hyperchaos-chaos" the trajectory is a generalized memory GM. With regard to tracking control, it is carried low exposure to hybrid noise $N$. Fractal dimensions:

$d_{f}(R-F)=1.2708$,

$d_{f}($ chen $)=1.3710$,

$\Delta=0.1002$.

Poincare average return time is:

$\langle\tau\rangle_{R-F}=4.0159,\langle\tau\rangle_{\text {chen }}=4.3279$.

Effects of the "proximity" define as:

$H\left(\langle\tau\rangle_{R-F},\langle\tau\rangle_{\text {chen }}\right) \leq \tilde{\varepsilon} \Leftrightarrow\langle\tau\rangle_{R-F} \subset\langle\tau\rangle_{\text {chen }}+\tilde{\varepsilon}$,

where $\tilde{\varepsilon}=0.3121$.

Examples of graphic images transformation results using the above-described algorithms over time series of chaotic and hyper-chaotic systems are shown in Fig. 4. 

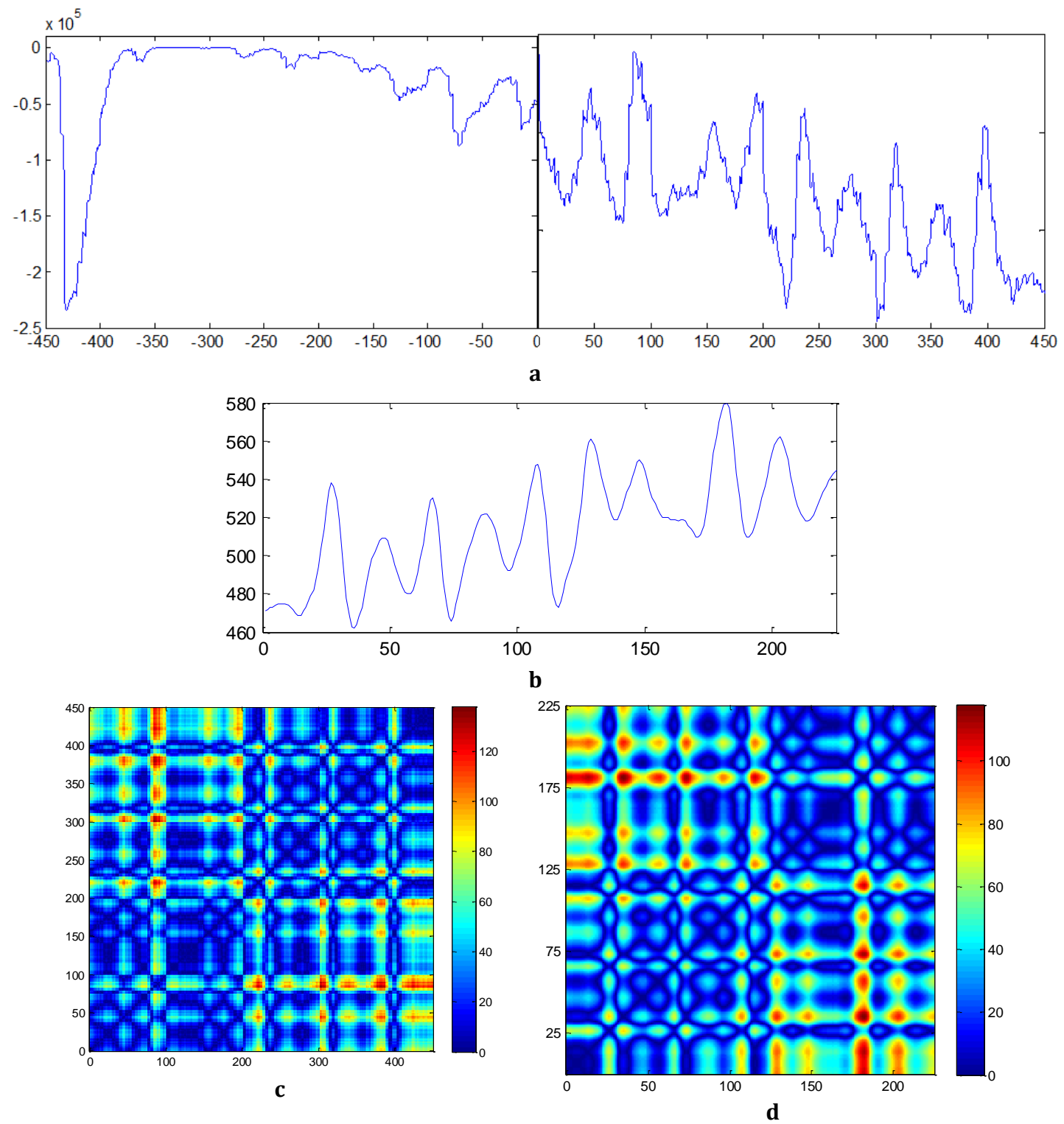

Fig. 3: Sequence of reconstruction of the signal according to the scheme "SSA-lifting scheme" and their recurrence diagrams: $a, b$ - semi-trajectories of chaos; c, d - Poincare diagrams

\section{Conclusion}

It is important to note the non-traditional model of the transition and recurrence processes and evolution of its properties using the generalized Poincare return time spectrum. The results obtained can be used in the transition process in chaotic and hyperchaotic fractional systems. In shows a complete picture of the transient type "hyperchaoschaos-chaos".

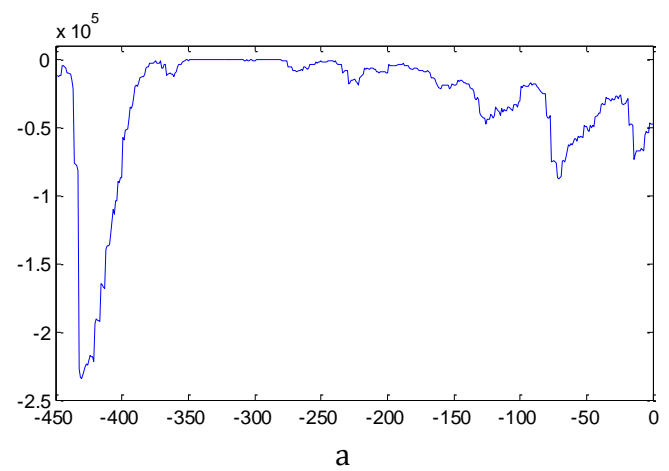

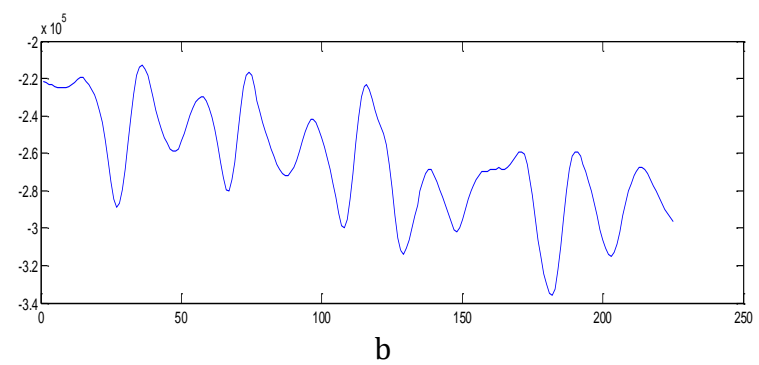

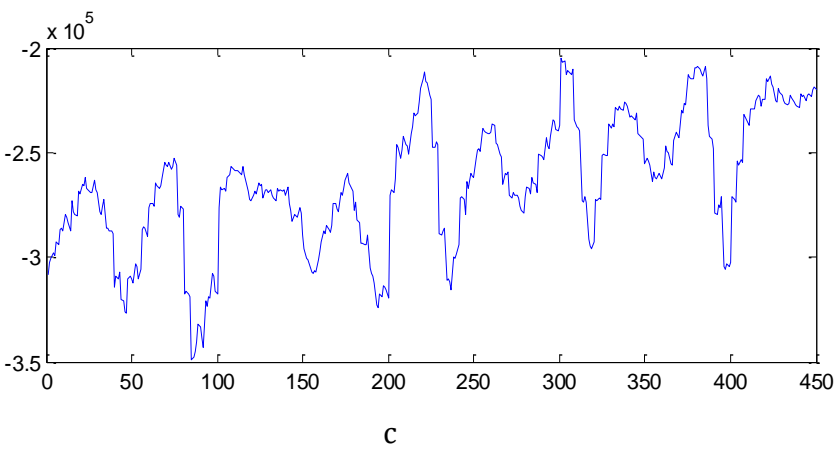



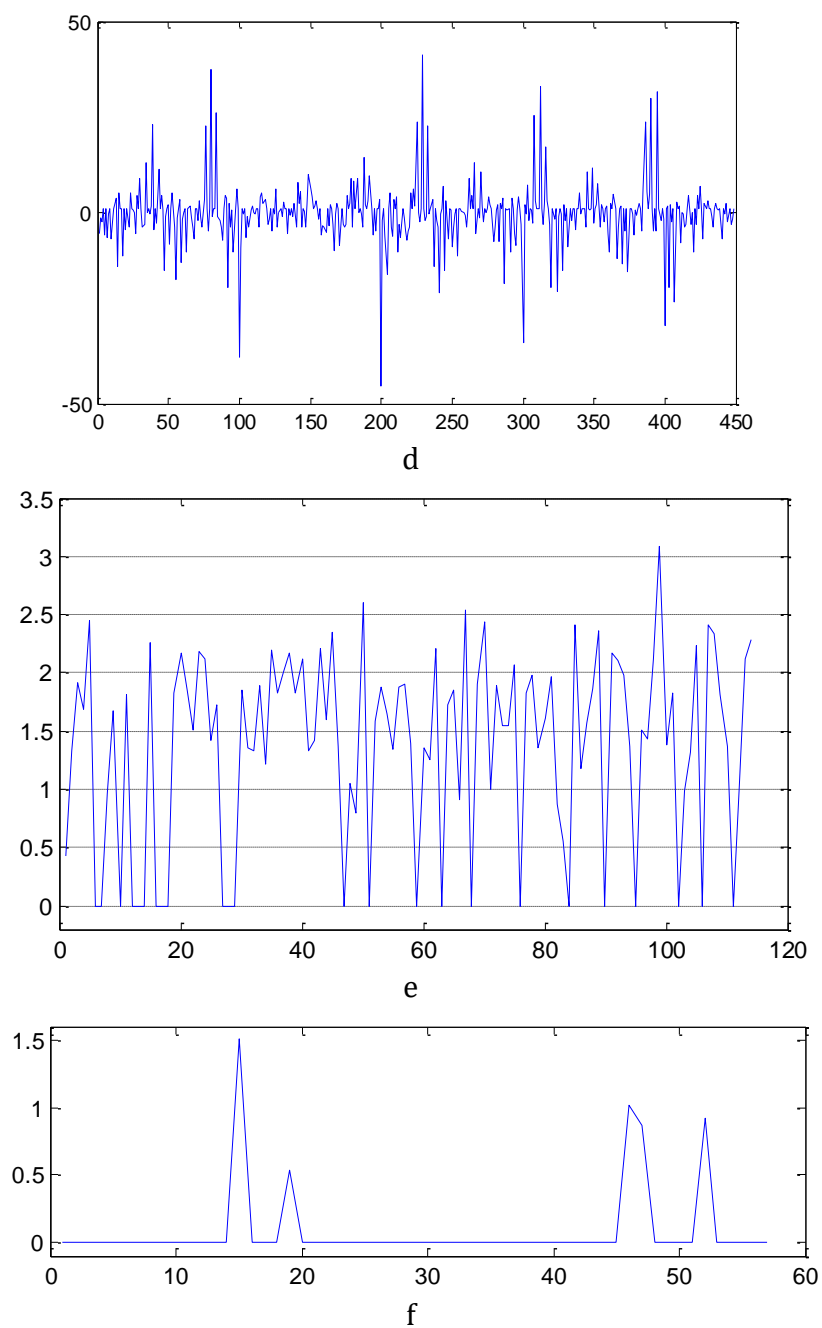

Fig. 4: Gallery of graphic images showing the stages of the time series of chaotic and hyper-chaotic systems:

a, b, c- transition trajectory of Tsallis entropy; Lyapunov indices: $d$ - stability, e- exponent, f-dimension

\section{References}

Afrajmovich V, Ugalde Je, and Urias H (2011). Fraktalnye razmernosti dlya vremen vozvrashheniya Puankare. Izhevskij Institut Kompyuternyx Issledovanij, Moscow, Russia.

Altmann EG and Tél T (2008). Poincaré recurrences from the perspective of transient chaos. Physical Review Letters, 100(17): 174101. https://doi.org/10.1103/PhysRevLett.100. 174101

Brox D (2017). The Riemann Hypothesis and Emergent Phase Space. Journal of Modern Physics, 8(4), Article ID: 74714, 24 pages. https://doi.org/10.4236/jmp.2017.84030

Butkovskiy AG, Postnov SS, and Postnova EA (2013). Drobnoe integrodifferentsialnoe ischislenie i ego prilozheniya $\mathrm{v}$ teorii upravleniya. I Matematicheskie Osnovyi i Problema Interpretatsii, AiT, 4: 3-42.

Chirikov BV (1979). A universal instability of many-dimensional oscillator systems. Physics reports, 52(5): 263-379.

Grigorenko I and Grigorenko E (2003). Chaotic dynamics of the fractional Lorenz system. Physical Review Letters, 91(3): 034101. https://doi.org/10.1103/PhysRevLett.91.034101

Ibedou I and Miyata T (2008). The theorem of PontrjaginSchnirelmann and approximate sequences. New Zealand Journal of Mathematics, 38: 121-128.
Ismailov BI (2016). Poincare recurrence in open systems. Journal of Multidisciplinary Engineer in Science and Technology, 3(9): 5565-5569.

Kusch HA and Ottino JM (1992). Experiments on mixing in continuous chaotic flows. Journal of Fluid Mechanics, 236: 319-348.

Lahlou TA and Oppenheim AV (2014). Spectral representation of transient signals. In the IEEE Global Conference Signal and Information Processing, IEEE, Atlanta, USA: 660-663. https://doi.org/10.1109/GlobalSIP.2014.7032200

Lekien F, Shadden SC, and Marsden JE (2007). Lagrangian coherent structures in n-dimensional systems. Journal of Mathematical Physics, 48(6): 065404. https://doi.org/10.1063/1.2740025

Leonov GA and Kuznetsov NV (2011). Analytical-numerical methods for investigation of hidden oscillations in nonlinear control systems. IFAC Proceedings Volumes, 44(1): 24942505.

Liu J and Teel AR (2014). Hybrid systems with memory: modelling and stability analysis via generalized solutions. IFAC Proceedings Volumes, 47(3): 6019-6024.

Marwan N, Romano MC, Thiel M, and Kurths J (2007). Recurrence plots for the analysis of complex systems. Physics Reports, 438(5): 237-329.

Nigmatullin RR (1992). Drobnyj integral. Teoreticheskaya i matematicheskaya fizika, 90(3): 354-367.

Norris JR (1998). Markov chains. Cambridge University Press, Cambridge, UK.

Riesz M (1949). L'intégrale de Riemann-Liouville et le problème de Cauchy. Acta mathematica, 81(1): 1-222.

Shadden SC (2011). Lagrangian coherent structures. In: Grigoriev $\mathrm{R}$ (Ed.), Transport and mixing in laminar flows: from Microfluidics to Oceanic Currents: 59-89. Wiley-VCH, Weinheim, Germany.

Sierociuk D and Ziubinski P (2014). Fractional order estimation schemes for fractional and integer order systems with constant and variable fractional order colored noise. Circuits, Systems, and Signal Processing, 33(12): 3861-3882.

Tarasov VE (2010). Fractional dynamics of open quantum systems. In: Fractional Dynamics. Nonlinear Physical Science: pp.467-490. Springer, Berlin, Heidelberg, Germany. https://doi.org/10.1007/978-3-642-14003-7_20

Teo CHG (2012). Brownian motion and Liouville's theorem. Available online at: http://math.uchicago.edu/ may/ REU2012/REUPapers/Teo.pdf

Tsallis C and Tirnakli U (2010). Nonadditive entropy and nonextensive statistical mechanics-Some central concepts and recent applications. Journal of Physics: Conference Series, 201(1): 1-15.

Vaidyanathan S and Volos C (2017). Advances in memristors, memristive devices and systems. Springer International Publishing, Berlin, Germany.

Vladimirsky EI and Ismailov BI (2015). Synchronization, control and stability of fractional order hyper chaotic systems in the context of the generalized memory. International Journal of New Technology and Research, 1(8): 42-48.

Vladimirsky EI and Ismailov BI (2016). Fractional-order chaotic filter with generalized memory. International Journal of Contemporary Applied Sciences 3(4): 46-61.

Weng T, Small M, Zhang J, and Hui P (2015). Lévy walk navigation in complex networks: A distinct relation between optimal transport exponent and network dimension. Scientific Reports. https://doi.org/ 10.1038/srep17309

Zaslavsky GM (2002). Chaos, fractional kinetics, and anomalous transport. Physics Reports, 371(6): 461-580. 